\title{
A PROBLEM CONNECTED WITH THE ZEROS OF RIEMANN'S ZETA FUNCTION
}

\author{
P. B. BRAUN AND A. ZULAUF
}

\begin{abstract}
Estimates are given for the number of zeros of $\operatorname{Re}\left\{\pi^{-s / 2} \Gamma(s / 2) \zeta(s)\right\}$ and $\operatorname{Im}\left\{\pi^{-s / 2} \Gamma(s / 2) \zeta(s)\right\}$ with $0<\operatorname{Im} s<T$, and fixed $\operatorname{Re} s$ inside the critical strip.
\end{abstract}

Let $f(s)=\pi^{-s / 2} \Gamma(s / 2) \zeta(s)$, where $\zeta$ is Riemann's zeta function. Suppose that $\lambda$ is a fixed real number such that $\frac{1}{2}<\lambda<1$, and let

$$
R(t)=\operatorname{Re} f(\lambda+i t), \quad I(t)=\operatorname{Im} f(\lambda+i t) .
$$

Finally let $N_{R}(\lambda, T)$ and $N_{I}(\lambda, T)$ be the number of zeros of $R(t)$ and $I(t)$, respectively, in the interval $0<t<T$, multiple zeros being counted according to their multiplicity.

Berlowitz [1] proved that $N_{R}(\lambda, T)$ and $N_{I}(\lambda, T)$ are unbounded as $T \rightarrow \infty$. Berndt [2] improved on this result by showing that there exists a positive constant $A$ such that, for all sufficiently large $T$,

$$
N_{R}(\lambda, T)>A T, \quad N_{I}(\lambda, T)>A T .
$$

The object of this paper is to obtain the following further improvement.

THEOREM. ${ }^{1}$ For every $\lambda$ such that $\frac{\lambda}{2}<\lambda<1$, there exists a positive constant $A$ such that, for all sufficiently large $T$,

$$
N_{R}(\lambda, T) \geqq(1 / 2 \pi) T \log T-A T, \quad N_{I}(\lambda, T) \geqq(1 / 2 \pi) T \log T-A T .
$$

Proof. We shall prove the Theorem for $N_{R}(\lambda, T)$; the proof for $N_{I}(\lambda, T)$ is the same, except for obvious changes of notation. Let $\frac{1}{2}<\lambda<1$, $T>0$. Without loss of generality we may assume that $T$ is not the imaginary part of a zero of $\zeta(s)$.

Let $L$ be the straight line segment joining the points $\lambda$ and $\lambda+i T$ modified, if need be, by small semicircular indentations so that any zeros of $\zeta(s)$ with real part $\lambda$ lie to the left of $L$. It is obvious that the number of

Received by the editors September 20, 1971.

AMS 1969 subject classifications. Primary 1041.

Key words and phrases. Riemann zeta function.

${ }^{1}$ Substantially the same result was obtained by N. Levinson in a paper, On theorems of Berlowitz and Berndt, which appeared in J. Number Theory 3 (1971), 502-504, shortly after our paper had been submitted. 


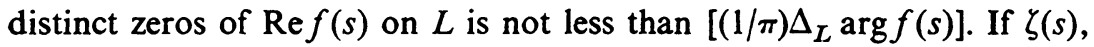
and hence $f(s)$, has a zero of order $n$ at $\lambda+i t_{0}$ then $R(t)$ has a zero of the same or higher order at $t_{0}$; on the other hand, if $\gamma$ be the semicircular detour by which $L$ avoids $\lambda+i t_{0}$, then $\operatorname{Re} f(s)$ has at most $n+1$ distinct zeros on $\gamma$, since

$(d / d \theta) \arg f\left(\lambda+i t_{0}+\delta e^{i \theta}\right)=\operatorname{Im}\left\{\left(f^{\prime} / f\right)\left(\lambda+i t_{0}+\delta e^{i \theta}\right) i \delta e^{i \theta}\right\}=n+O(\delta)$ so that $\arg f(s)$ increases monotonically by $n \pi+O(\delta)$ on $\gamma$, it being assumed that the radius $\delta$ of $\gamma$ is sufficiently small. Hence it follows easily that

$$
N_{R}(\lambda, T) \geqq\left[(1 / \pi) \Delta_{L} \arg f(s)\right]-N_{0}(\lambda, T),
$$

where $N_{0}(\lambda, T)$ is the number of zeros of $\zeta(s)$ on the straight line segment from $\lambda$ to $\lambda+i T$. It is well known that $N_{0}(\lambda, T)=O(T)$, and it is easily shown (cf. the Lemma below) that

$$
\Delta_{L} \arg f(s)=\frac{1}{2} T \log T+O(T) .
$$

By (1) and (2) the Theorem is thus established.

LEMMA. If $L$ is defined as in the proof of the Theorem above, then (2) holds.

Proof. Let $L^{\prime}$ be the contour which is symmetric to $L$ about the line of real part $\frac{1}{2}$; and let $C$ be the simple closed contour which goes along $L$ from $\lambda$ to $\lambda+i T$, then straight from $\lambda+i T$ to $1-\lambda+i T$, then along $L^{\prime}$ from $1-\lambda+i T$ to $1-\lambda$, and finally straight from $1-\lambda$ back to $\lambda$.

It is well known that the number of zeros of $f(s)$ (or, equivalently, of $\zeta(s))$ inside $C$ is $(1 / 2 \pi) T \log T+O(T)$. Hence

$$
\Delta_{C} \arg f(s)=T \log T+O(T) .
$$

But $f(s)$ is real on the real axis, and $f(1-s)=f(s)$ and $f(\bar{s})=\overline{f(s)}$ for all $s$. Hence

$$
\Delta_{C} \arg f(s)=2 \Delta_{L} \arg f(s)+2 \Delta_{K} \arg f(s),
$$

where $K$ is the straight line segment from $\lambda+i T$ to $\frac{1}{2}+i T$. But

$$
\begin{aligned}
& \Delta_{K} \arg \left(\pi^{-s / 2}\right)=\Delta_{K} \operatorname{Im} \log \left(\pi^{-s / 2}\right)=0, \\
& \Delta_{K} \arg \Gamma(s / 2)=\Delta_{K} \operatorname{Im} \log \Gamma(s / 2)=O(1),
\end{aligned}
$$

as may be seen by Stirling's formula, and

$$
\Delta_{K} \arg \zeta(s)=-\int_{1 / 2+i T}^{\lambda+i T} \operatorname{Im}\left(\zeta^{\prime}(s) / \zeta(s)\right) d s=O(\log T),
$$


as may be shown by the method given in Davenport [3, pp. 103-104]. Hence

$$
\Delta_{K} \arg f(s)=O(\log T) .
$$

The desired result (2) now follows from (3), (4), and (5).

By using improved estimates for $N_{0}(\lambda, T)$ and for the number of zeros of $\zeta(s)$ inside $C$ (see Titchmarch [4, Theorems 9.4 and 9.19]), it can easily be seen that the term $A T$ in our Theorem may be replaced by

$$
(1 / 2 \pi)(1+\log (2 \pi)) T+o(T) .
$$

\section{REFERENCES}

1. B. Berlowitz, Extensions of a theorem of Hardy, Acta Arith. 14 (1967/68), 203-207. MR 37 \#1324.

2. B. C. Berndt, On the zeros of the Riemann zeta-function, Proc. Amer. Math. Soc. 22 (1969), 183-188. MR 39 \#4104.

3. H. Davenport, Multiplicative number theory, Lectures in Advanced Math., no. 1, Markham, Chicago, Ill., 1967. MR 36 \#117.

4. E. C. Titchmarch, The theory of the Riemann zeta function, Clarendon Press, Oxford, 1951.

Department of Mathematics, University of Waikato, Hamilton, New Zealand 